\title{
Cardioprotective Effect of High-Intensity Aerobic Interval Training against Adriamycin-Induced Cardiac Toxicity in Rats
}

\author{
Khadijeh Ebrahimi (iD) ${ }^{1,}$, Siroos Choobineh (iD ${ }^{2,{ }^{* *}}$, Rahman Soori (iD ${ }^{2}$ and Reza Badalzadeh (iD ${ }^{3}$ \\ ${ }^{1}$ Department of Physical Education and Sport Sciences, Marand Branch, Islamic Azad University, Marand, Iran \\ ${ }^{2}$ Department of Exercise Physiology, Faculty of Physical Education and Sport Sciences, University of Tehran, Tehran, Iran \\ ${ }^{3}$ Molecular Medicine Research Center and Drug Applied Research Center, Tabriz University of Medical Sciences, Tabriz, Iran \\ Corresponding author: Department of Physical Education and Sport Sciences, Marand Branch, Islamic Azad University, Postal code: 5418916571, Marand, Iran. Email: \\ ebrahimi_kh@ut.ac.ir \\ ${ }^{* *}$ Corresponding author: Associate Professor of Sports Physiology, Department of Exercise Physiology, Faculty of Physical Education and Sport Sciences, University of Tehran, \\ Tehran, Iran. Fax: +98-2161118844, Email: choobineh@ut.ac.ir
}

Received 2018 September 02; Revised 2019 February 02; Accepted 2019 February 04.

\begin{abstract}
Background: Adriamycin (ADR) is a useful drug for the treatment of hematologic malignancies and solid tumors. However, its clinical uses are limited due to dose-dependent cardiac toxicity. Contrary to moderate endurance training, there is little research about the protective role of high-intensity aerobic interval training (HIIT) against the ADR-induced cardiac toxicity and its mechanisms. Objectives: The present study aimed to investigate the protective effect of HIIT against ADR-induced cardiac toxicity in the left ventricle of rats by assessment of the serum biomarkers of cardiac injury (LDH and CK-MB).

Methods: Twenty-four male Wistar rats were randomly assigned into four groups ( $\mathrm{n}=6 /$ group): (1) Control; (2) ADR (20 mg/kg.bw); 3) HIIT ( 7 sets of 4 minutes intervals at $80 \%-90 \% \mathrm{VO}_{2}$ max interspersed with 3 minutes periods of $65 \%-75 \% \mathrm{VO}_{2} \mathrm{max}$, for 8 weeks), and (4) HIIT + ADR. The ELISA method was used for measuring the serum levels of biomarkers. Statistical differences were analyzed using one-way analysis of variance followed by Tukey's post hoc test for multiple comparisons $(\alpha<0.05)$.

Results: Based on the results, ADR-induction resulted in a significant increase in the LDH and CK-MB levels compared to the ADR group $(\mathrm{P}<0.05)$. Also, the HIIT per se insignificantly increased the levels of LDH and CK-MB compared to the control group. However, the HIIT before ADR-induction resulted in a significant decrease in the LDH and CK-MB levels compared to the ADR group ( $<<0.05)$. Conclusions: Therefore, the HIIT can be a proper non-prescriptive strategy for preventing ADR-induced cardiotoxicity via reducing the serum biomarkers of cardiac injury.
\end{abstract}

Keywords: Interval-Training, Adriamycin, Cardiac Toxicity, Cardiac Biomarkers

\section{Background}

Adriamycin (ADR), as one of the most common anticancer anthracyclines, is used widely to treat hematologic malignancies and solid tumors such as acute leukemia, bone/soft tissue sarcoma, and many other malignant neoplasms (1). However, its prescription has been limited due to cardiac toxicity (irreversible dose-dependent cardiac damage), during or even years after the treatment (2). Although the molecular mechanisms of ADR-induced cardiac toxicity are not yet fully understood, excess production of reactive oxygen species (ROS) and mitochondrial dysfunction have been suggested as well-accepted mechanisms (3). The level of ADR-induced ROS production in the heart tissue is ten-fold higher than that in other tissues such as liver, kidney, and spleen, which shows the sensitivity of heart tissue to this drug (4).
The cardiac biomarkers are released into the bloodstream when the heart is damaged (2). Of the many enzymes used for the detection of cardiac dysfunction, lactate dehydrogenase (LDH) and creatine kinase (CK) have gained wide clinical approval $(1,2)$. The results of various studies have shown that the serum biomarkers of cardiac injury increase following ADR-treatment in the blood $(5,6)$. In one study, Venkatesan examined the effect of intraperitoneal (i.p.) injection of ADR (30 mg/kg.bw) on cardiac enzymes in rats, and stated that the serum levels of CK-MB and LDH were significantly increased compared to the control group (5). Also, Shirinbayan and Roshan reported that ADR-induction led to an increase in the serum level of biomarkers of cardiac injury, including LDH, CKMB, troponin-I (cTnI), alanine aminotransferase (ALT) and aspartate aminotransferase (AST) in rats (7). Therefore, the primary diagnosis of ADR-induced cardiac toxicity could

Copyright (c) 2019, Journal of Clinical Research in Paramedical Sciences. This is an open-access article distributed under the terms of the Creative Commons Attribution-NonCommercial 4.0 International License (http://creativecommons.org/licenses/by-nc/4.0/) which permits copy and redistribute the material just in noncommercial usages, provided the original work is properly cited. 
provide the proper prevention strategy $(2,8)$.

Older cancer patients often die of heart failure during the ADR-treatment, while child cancer survivors live long enough to face the long-term cardiac toxicity consequences (3). More than a quarter of ADR-treated patients have died of the cardiovascular diseases (9). Therefore, different strategies should be taken to prevent ADRinduced secondary cardiovascular side-effects. Moderate endurance exercises, as one of the proper protective strategies, can protect the heart against the ADR-induced cardiac toxicity $(10,11)$.

Furthermore, high-intensity aerobic interval training (HIIT), including repetitive periods of high-intensity training with periods of rest or low-intensity training (12), has a positive effect on the cardiovascular system in patients (13). HIIT can improve the stability of cardiomyocytes energy metabolism in patients with chronic heart failure via upregulation of the AMPK pathway (14). Also, recent research has suggested that cardiovascular adaptation associated with HIIT in some cases is more than that of moderate endurance exercises (15). For instance, the HIIT-induced $\mathrm{VO}_{2}$ max development is twice as high as that of endurance exercise (16). It has been shown that HIT prior to and during ADR-treatment could reduce cardiovascular sideeffects by increasing the antioxidant enzyme content in the rats' heart tissue (17). However, few articles have addressed the protective role of HIIT against the ADR-induced cardiac toxicity and its cellular mechanisms.

\section{Objectives}

The purpose of this study was to investigate the protective effect of HIIT against ADR-induced cardiac toxicity in the left ventricle of rats by assessing the serum biomarkers of cardiac injury (LDH and CK-MB).

\section{Methods}

\subsection{Animals}

Twenty-four male Wistar rats (3 weeks old) were purchased from the Laboratory Animal Research Center of Tabriz University of Medical Sciences. In order to adapt to the new environment, the rats were kept for a week at room under controlled temperature (of $21 \pm 2{ }^{\circ} \mathrm{C}$ ) with free access to food and water under the 12-hour light/dark cycle. All rats were initially familiarized with a rodent's treadmills (3 sessions, $10 \mathrm{~m} / \mathrm{min}, 15$ minutes) before randomization (Table 1). Then, the animals were divided into one of four groups randomly ( $n=6$ per groups): (1) sedentary control (control), (2) sedentary adriamycin-treated (ADR), (3) exercise trained (HIIT), and exercise-trained, treated with ADR
(HIIT + ADR). All experimental procedures were reviewed in Research Ethics Committee of Sport Sciences Research Institute and were approved according to Ethics Standards in Research of the Ministry of Science, Research and Technology, (IR.SSRI.REC.1397.311).

\begin{tabular}{lc}
\hline Table 1. Timeline for Applying Variables & Timeline \\
\hline Variable & One week \\
\hline $\begin{array}{l}\text { Maintenance in the laboratory } \\
\text { environment }\end{array}$ & One week \\
Familiar with the treadmill & Eight weeks \\
HIIT & Immediately after the last training \\
ADR-treatment & session \\
\hline Blood sampling & 72 hours after ADR-injection \\
\hline Abbreviations: ADR, adriamycin; HIIT, high-intensity aerobic interval training.
\end{tabular}

\subsection{HIIT Protocol}

Exercise protocols were developed based on a previous study (18). Breifly, HIIT was performed 1 hour/day, 5 days/week for 8 weeks in the morning from saturday to wednesday. HIT protocol included three steps: (1) Warmup: 10 minutes warm-up with 50\% - 55\% $\mathrm{VO}_{2} \max$, (2) Main body: 7 sets of intervals with 4 minutes at $80 \%-90 \%$ $\mathrm{VO}_{2}$ max interspersed with 3 minutes periods of $65 \%-75 \%$ $\mathrm{VO}_{2}$ max, (3) cool-down: 1 minute cool down period with $50 \%-55 \% \mathrm{VO}_{2} \mathrm{max}$. Because we did not have instruments such as respiratory gas analyzers for estimating $\mathrm{VO}_{2}$ max directly, we indirectly estimated the intensity of animal exercise according to the speed of movement at the beginning of every 2 weeks (there is a strong correlation between the running speed and $\mathrm{VO}_{2} \max$ ) (19).

\subsection{ADR Injection and Biomarkers Measurement}

Immediately after the last HIIT session, animals received a cumulative dose of ADR hydrochloride (20 $\mathrm{mg} / \mathrm{kg}$.bw, i.p.). Groups without ADR-treatment received a similar amount of saline. Seventy-two hours after ADR or saline-injection, each rat was anesthetized using an i.p. injection of xylazine-ketamine combination (90 and 10 $\mathrm{mg} / \mathrm{kg}$, respectively). Blood samples were taken directly from the left ventricle of the rats and immediately centrifuged at $3000 \mathrm{rpm}$ for 10 minutes. Afterward, serum was separated from the plasma and kept at $70^{\circ} \mathrm{C}$ for measuring LDH and CK-MB levels. The LDH and CK-MB levels, indicators of cardiac damage (20), were measured using spectrophotometric procedures with available kits (Roche Diagnostics, Germany) according to the manufacturer's instructions. The enzyme values are reported as $\mathrm{U} / \mathrm{L}$. 


\subsection{Statistical Analysis}

The results are expressed as the mean \pm standard deviation (SD) for each experimental group. Statistical differences were analyzed using one-way analysis of variance followed by Tukey's post hoc test for multiple comparisons in SPSS version 23 software $(\alpha<0.05)$.

\section{Results}

Based on the results, ADR-induction could significantly increase LDH and CK-MB levels in the serum samples compared to the control group $(\mathrm{P}<0.05)$. Also, HIIT per se insignificantly increased the serum levels of LDH and CK-MB compared to the control group. However, HIIT before ADRtreatment resulted in a significant decrease in the $\mathrm{LDH}$ and CK-MB levels compared to the ADR group $(\mathrm{P}<0.05)$. Therefore, HIIT could be a proper non-prescriptive strategy for preventing of ADR-induced cardiac toxicity (Figure 1).

\section{Discussion}

In this study, ADR-treatment significantly increased the serum biomarkers of cardiac injury. The present study also revealed that eight weeks of HIIT before ADRtreatment could significantly decrease the serum levels of LDH and CK-MB.

The results of this study are similar to those reported by previous researchers $(19,20)$ that ADR-induction leads to cardiac toxicity along with the increased levels of cardiac injury biomarkers in rats. Consistently, Venkatesan stated that ADR-treatment led to an increase in the serum levels of CK and LDH in rats (5). Yagmurca et al. also noted that the activity and levels of AST, LDH and CK-MB, as well as the heart rate and blood pressure were significantly increased in the mice treated with acute ADR-injection (20 mg/kg.bw i.p.) compared to the healthy animals (18). Another study by Pizarro et al. revealed that chronic ADRinduction led to a 50\% increase in the serum LDH levels and cardiomyocytes necrosis after 24 hours of treatment (21). Also, Nimbal and Koti who studied the effect of some kinds of vitamin supplements on ADR-induced cardiac toxicity reported that acute ADR-induction results in a significant increase in the serum LDH, CK-MB and cTnI levels (22). Mantawy et al. also stated that chronic ADR-induced cardiac toxicity (15 mg/kg.bw i.p.) resulted in a significant increase in the serum LDH and CK-MB levels in rats (23). In agreement with our results, a significant increase was observed in the serum levels of LDH and creatine phosphokinase (CPK) in rats after 72 hours of the ADR-tratment (chronic injection, $10 \mathrm{mg} / \mathrm{kg} . \mathrm{bw}$ i.p.) (24).
There is increasing evidence of the protective role of exercise training against ADR-induced cardiac toxicity (25). Shirinbayan and Roshan suggested that endurance exercise for three weeks before ADR-induction could remarkably increase cardioprotective markers such as heat shock protein (HSP70 kDa) and superoxide dismutase (SOD), and decrease cardiac and oxidative damage (MDA, CK, CK-MB and $\mathrm{CK} / \mathrm{CPK}-\mathrm{MB}$ ) in rat serum (7). Another study showed that eight weeks of endurance exercise prior to chronic induction of ADR could reduce cardiac toxicity by reducing serum LDH levels (26). Kirkham et al. suggested that shortterm endurance exercise before treatment of cancer patients with ADR could reduce serum cTnT levels (27). In general, previous reports indicated that endurance exercise could protect the cardiomyocytes against the ADR-induced cardiac toxicity.

Notably, HIIT could also prevent heart damage. For example, studies suggest that HIIT could protect the heart against ischemia reperfusion (IR) injury by reducing the plasma LDH and CK-MB activity, as well as the myocardial infarct size (28). Furthermore, HIIT, compared to the endurance exercise, resulted in a similar or even greater improvement of cardiovascular system in patients (29-31). However, there are few studies about the preventative role of HIIT against the ADR-induced cardiac toxicity. Recently, Jarrett et al. revealed that HIIT before ADR-treatment could protect the heart via increasing the antioxidant enzyme content (17). This finding is also in agreement with our result that eight weeks of HIIT could protect the heart against the ADR-induced cardiac toxicity by decreasing the serum levels of LDH and CK-MB.

There is strong evidence that HIIT triggers higher metabolic signaling (accumulation of intracellular lactate production, AMP, ADP, AMPK, as well as the reliance on carbohydrate oxidation) than moderate intensity endurance training (32). Nevertheless, the molecular mechanisms of the protective effect of HIIT against the ADR-induced cardiac disorders have not been thoroughly investigated. It has been reported that HIIT after heart failure could improve cardiac function by inhibition of ADR-induced changes in the mitochondrial dynamics and biogenesis, antioxidant content, AMPK, and ERK1/2-JNK-P53 signaling pathways (33). ADR-treatment is reported to increase ROS content (34) and bioenergy metabolism impairement (by AMPK signaling inhibition, and mTOR signaling activation) in cardiomyocytes (35). Wang et al. have shown that HIIT per se could upregulate the energy metabolism of cardiomyocytes, and thereby improve the energy production in patients with chronic heart failure (14). Therefore, antioxidants, mitochondrial dynamics, biogenesis, and AMPK signaling pathways could be the potential molecular mechanisms of HIIT in protecting cardiomyocytes against 
A

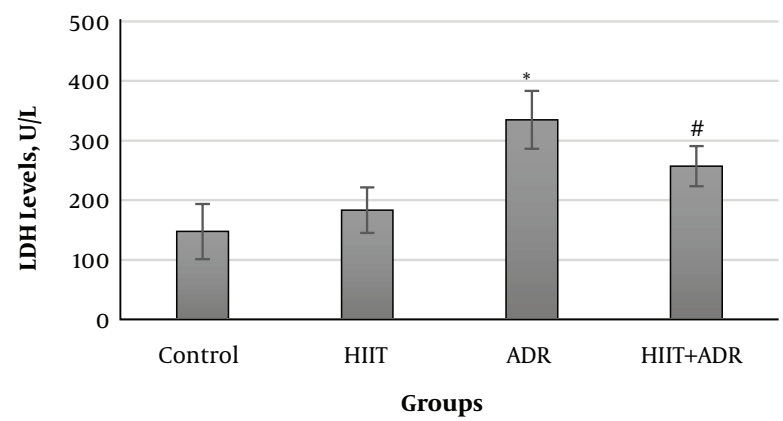

B

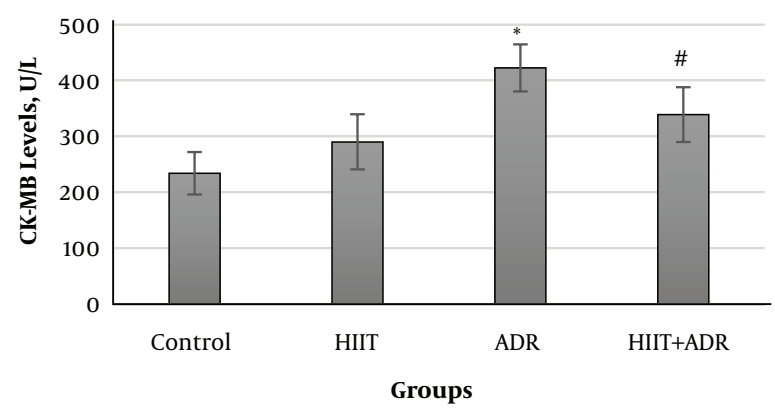

Figure 1. A, LDH and B, CK-MB levels in the blood of the rats of the study groups. *Compared to the control group. \#Compared to the ADR group ( $\alpha<0.05)$.

the ADR-induced toxicity.

\subsection{Conclusions}

Cardiac toxicity due to chronic induction of ADR (20 $\mathrm{mg} / \mathrm{kg}$.bw i.p.) in rats is associated with a significant increase in the serum biomarkers of cardiac injury after 72 hours. However, HIIT prior to ADR-induction can lead to a significant decrease in the serum levels of LDH and CK-MB. Therefore, HIIT can be a proper protective strategey against ADR-induced cardiac toxicity.

\section{Footnotes}

Authors' Contribution: Study concept and design: Khadijeh Ebrahimi; analysis and interpretation of data: Reza Badalzadeh; drafting of the manuscript, critical revision of the manuscript for important intellectual content: Khadijeh Ebrahimi and Siroos Choobineh; statistical analysis: Rahman Soori.

Conflict of Interests: The authors declare no conflict of interest regarding the compilation or publication of this article.

Ethical Approval: All experimental procedures were reviewed in Research Ethics Committee of Sport Sciences Research Institute and were approved according to ethics standards in research of the Ministry of Science, Research and Technology, (IR.SSRI.REC.1397.311).

Funding/Support: This research received no grant from any funding agency in the public, commercial or not-forprofit sectors.

\section{References}

1. Alkuraishy HM, Al-Gareeb AI, Al-hussaniy HA. Doxorubicin induced cardiotoxicity: Molecular mechanism and protection by conventional drugs and natural products. Int J Clin Oncol Cancer Res. 2017;2(2):31-44.
2. Cao L, Zhu W, Wagar EA, Meng QH. Biomarkers for monitoring chemotherapy-induced cardiotoxicity. Crit Rev Clin Lab Sci. 2017;54(2):87-101. doi: 10.1080/10408363.2016.1261270. [PubMed: 28013560].

3. Chen JJ, Wu PT, Middlekauff HR, Nguyen KL. Aerobic exercise in anthracycline-induced cardiotoxicity: A systematic review of current evidence and future directions. Am J Physiol Heart Circ Physiol. 2017;312(2):H213-22. doi: 10.1152/ajpheart.00646.2016. [PubMed: 27923793].

4. Diamanti J, Mezzetti B, Giampieri F, Alvarez-Suarez JM, Quiles JL, Gonzalez-Alonso A, et al. Doxorubicin-induced oxidative stress in rats is efficiently counteracted by dietary anthocyanin differently enriched strawberry (Fragaria x ananassa Duch.). J Agric Food Chem. 2014;62(18):3935-43. doi: 10.1021/jf405721d. [PubMed: 24580025].

5. Venkatesan N. Curcumin attenuation of acute adriamycin myocardial toxicity in rats. $B r J$ Pharmacol. 1998;124(3):425-7. doi: 10.1038/sj.bjp.0701877. [PubMed: 9647462]. [PubMed Central: PMC1565424].

6. Horino N, Kobayashi Y, Usui T. Elevation of lipid peroxide in children treated with a combination of chemotherapeutic agents including doxorubicin. Acta Paediatr Scand. 1983;72(4):549-51. doi: 10.1111/j.16512227.1983.tb09769.x. [PubMed: 6624430].

7. Shirinbayan V, Roshan VD. Pretreatment effect of running exercise on HSP70 and DOX-induced cardiotoxicity. Asian Pac J Cancer Prev. 2012;13(11):5849-55. doi: 10.7314/APJCP.2012.13.11.5849. [PubMed: 23317268].

8. Cardinale D, Scherrer-Crosbie M. The role of biomarkers. Anticancer treatments and cardiotoxicity. Elsevier; 2017. p. 281-90. doi: 10.1016/b978-0-12-802509-3.00024-8.

9. Swain SM, Whaley FS, Ewer MS. Congestive heart failure in patients treated with doxorubicin: A retrospective analysis of three trials. Cancer. 2003;97(11):2869-79. doi:10.1002/cncr.11407. [PubMed: 12767102].

10. Maia TN, Araujo GBR, Teixeira JAC, Alves Junior ED, Dias KP. Cardiotoxicity of doxorubicin treatment and physical activity: A systematic review. Int J Cardiovasc Sci. 2017;30(1):70-80. doi: 10.5935/23594802.20170004.

11. Kavazis AN, Smuder AJ, Powers SK. Effects of short-term endurance exercise training on acute doxorubicin-induced FoxO transcription in cardiac and skeletal muscle. J Appl Physiol (1985). 2014;117(3):223-30. doi: 10.1152/japplphysiol.00210.2014. [PubMed: 24947024]. [PubMed Central: PMC4347740].

12. Gibala MJ, Jones AM. Physiological and performance adaptations to high-intensity interval training. Nestle Nutr Inst Workshop Ser. 2013;76:51-60. doi: 10.1159/000350256. [PubMed: 23899754]. 
13. Guiraud T, Nigam A, Gremeaux V, Meyer P, Juneau M, Bosquet L. High-intensity interval training in cardiac rehabilitation. Sports Med. 2012;42(7):587-605. doi: 10.2165/11631910-000000000-00000. [PubMed: 22694349].

14. Wang L, Gao K, Wang D. Exercise training has restorative potential on myocardial energy metabolism in rats with chronic heart failure. Iran J Basic Med Sci. 2018;21(8):818-23. doi: 10.22038/IJBMS.2018.29294.7076. [PubMed: 30186569]. [PubMed Central: PMC6118078].

15. Zuhl M, Kravitz L. Hiit vs. continuous endurance training: Battle of the aerobic titans. IDEA Fit J. 2012;9(2):34-40.

16. Wisloff $\mathrm{U}$, Ellingsen $\mathrm{O}$, Kemi OJ. High-intensity interval training to maximize cardiac benefits of exercise training? Exerc Sport Sci Rev. 2009;37(3):139-46. doi: 10.1097/JES.0b013e3181aa65fc. [PubMed: 19550205].

17. Jarrett CL, D'Lugos AC, Mahmood TN, Gonzales RJ, Hale TM, Carroll CC, et al. Effect of high intensity exercise preconditioning and training on antioxidant enzymes in cardiomyocytes during doxorubicin treatment. FASEB J. 2016;30(1_supplement):lb601.

18. Yagmurca M, Fadillioglu E, Erdogan H, Ucar M, Sogut S, Irmak MK. Erdosteine prevents doxorubicin-induced cardiotoxicity in rats. Pharmacol Res. 2003;48(4):377-82. doi: 10.1016/S1043-6618(03)00185-3. [PubMed: 12902208].

19. Hoydal MA, Wisloff U, Kemi OJ, Ellingsen O. Running speed and maximal oxygen uptake in rats and mice: Practical implications for exercise training. Eur J Cardiovasc Prev Rehabil. 2007;14(6):753-60. doi: 10.1097/HJR.ob013e3281eacef1. [PubMed:18043295].

20. Kemp M, Donovan J, Higham H, Hooper J. Biochemical markers of myocardial injury. Br J Anaesth. 2004;93(1):63-73. doi: 10.1093/bja/aeh148. [PubMed: 15096441].

21. Pizarro M, Troncoso R, Martinez GJ, Chiong M, Castro PF, Lavandero S. Basal autophagy protects cardiomyocytes from doxorubicin-induced toxicity. Toxicology. 2016;370:41-8. doi: 10.1016/j.tox.2016.09.011. [PubMed: 27666003].

22. Nimbal SK, Koti BC. Effect of ethanolic extract fractions of boerhaavia diffusa in doxorubicin-induced myocardial toxicity in albino rats. $J$ Young Pharm. 2017;9(4):545-9. doi: 10.5530/jyp.2017.9.104.

23. Mantawy EM, El-Bakly WM, Esmat A, Badr AM, El-Demerdash E. Chrysin alleviates acute doxorubicin cardiotoxicity in rats via suppression of oxidative stress, inflammation and apoptosis. Eur J Pharmacol. 2014;728:107-18. doi:10.1016/j.ejphar.2014.01.065. [PubMed: 24509133].

24. El-Shitany NA, El-Haggar S, El-desoky K. Silymarin prevents adriamycin-induced cardiotoxicity and nephrotoxicity in rats. Food Chem Toxicol. 2008;46(7):2422-8. doi: 10.1016/j.fct.2008.03.033. [PubMed: 18487002].

25. Félix DNM. Effects of voluntary physical activity and endurance training on cardiac mitochondrial function of rats sub-chronically treated with doxorubicin [dissertation]. University of Porto; 2013.
26. Greufe SE. Effect of endurance exercise on the combination of streptoxotocin induced diabetes and doxorubicin [dissertation]. University of Northern Colorado; 2013.

27. Kirkham AA, Eves ND, Shave RE, Bland KA, Bovard J, Gelmon KA, et al. The effect of an aerobic exercise bout $24 \mathrm{~h}$ prior to each doxorubicin treatment for breast cancer on markers of cardiotoxicity and treatment symptoms: A RCT. Breast Cancer Res Treat. 2018;167(3):719-29. doi: 10.1007/s10549-017-4554-4. [PubMed: 29110150].

28. Rahimi M, Shekarforoush S, Asgari AR, Khoshbaten A, Rajabi H, Bazgir $B$, et al. The effect of high intensity interval training on cardioprotection against ischemia-reperfusion injury in wistar rats. EXCLI J. 2015;14:237-46. doi: 10.17179/excli2014-587. [PubMed: 26417361]. [PubMed Central: PMC4555214].

29. Wisloff U, Stoylen A, Loennechen JP, Bruvold M, Rognmo O, Haram PM, et al. Superior cardiovascular effect of aerobic interval training versus moderate continuous training in heart failure patients: A randomized study. Circulation. 2007;115(24):3086-94. doi: 10.1161/CIRCULATIONAHA.106.675041. [PubMed: 17548726].

30. Fu TC, Wang CH, Lin PS, Hsu CC, Cherng WJ, Huang SC, et al. Aerobic interval training improves oxygen uptake efficiency by enhancing cerebral and muscular hemodynamics in patients with heart failure. Int J Cardiol. 2013;167(1):41-50. doi: 10.1016/j.ijcard.2011.11.086. [PubMed: 22197120].

31. Villelabeitia-Jaureguizar K, Vicente-Campos D, Senen AB, Jimenez VH, Garrido-Lestache MEB, Chicharro JL. Effects of high-intensity interval versus continuous exercise training on post-exercise heart rate recovery in coronary heart-disease patients. Int J Cardiol. 2017;244:17-23. doi: 10.1016/j.ijcard.2017.06.067. [PubMed: 28648356].

32. MacInnis MJ, Gibala MJ. Physiological adaptations to interval training and the role of exercise intensity. J Physiol. 2017;595(9):291530. doi: 10.1113/JP273196. [PubMed: 27748956]. [PubMed Central: PMC5407969].

33. Jiang HK, Wang YH, Sun L, He X, Zhao M, Feng ZH, et al. Aerobic interval training attenuates mitochondrial dysfunction in rats postmyocardial infarction: Roles of mitochondrial network dynamics. Int J Mol Sci. 2014;15(4):5304-22. doi: 10.3390/ijms15045304. [PubMed: 24675698]. [PubMed Central: PMC4013565].

34. Octavia Y, Tocchetti CG, Gabrielson KL, Janssens S, Crijns HJ, Moens AL. Doxorubicin-induced cardiomyopathy: From molecular mechanisms to therapeutic strategies. J Mol Cell Cardiol. 2012;52(6):1213-25. doi: 10.1016/j.yjmcc.2012.03.006. [PubMed: 22465037].

35. Gratia S, Kay L, Potenza L, Seffouh A, Novel-Chate V, Schnebelen C, et al. Inhibition of AMPK signalling by doxorubicin: At the crossroads of the cardiac responses to energetic, oxidative, and genotoxic stress. Cardiovasc Res. 2012;95(3):290-9. doi: 10.1093/cvr/cvs134. [PubMed: 22461523]. 